\title{
New methods for solving a vertex p-center problem with uncertain demand-weighted distance: A real case study
}

\author{
Javad Nematian and Mir Ehsan Hesam Sadati*
}

Department of Industrial Engineering, University of Tabriz, P.O. Box 51666-14766, Tabriz, Iran

CHRONICLE ABSTRACT

\begin{tabular}{l}
\hline Article history: \\
Received July 112014 \\
Received in Revised Format \\
October 232014 \\
Accepted October 262014 \\
Available online \\
October 262014 \\
\hline Keywords: \\
Vertex p-center problem \\
Possibility theory \\
Fuzzy random variable \\
Fuzzy random chance-constrained \\
programming
\end{tabular}

\begin{abstract}
Vertex and p-center problems are two well-known types of the center problem. In this paper, a pcenter problem with uncertain demand-weighted distance will be introduced in which the demands are considered as fuzzy random variables (FRVs) and the objective of the problem is to minimize the maximum distance between a node and its nearest facility. Then, by introducing new methods, the proposed problem is converted to deterministic integer programming (IP) problems where these methods will be obtained through the implementation of the possibility theory and fuzzy random chance-constrained programming (FRCCP). Finally, the proposed methods are applied for locating bicycle stations in the city of Tabriz in Iran as a real case study. The computational results of our study show that these methods can be implemented for the center problem with uncertain frameworks.
\end{abstract}

\section{Introduction}

The facility location-allocation problem plays an important role in many firms and organizations. It is particularly suitable for telecommunication networks, emergency service systems, public services, etc. For a class of two-facility location-allocation problems with dense demand data, Murat et al. (2011) proposed an efficient allocation-based solution framework and specially they explained that previous results for the discrete demand case could be extended to problems with highly dense demand data. Kim et al. (2011) dealt with a physical access network design problem of fiber-to-the-home passive optical network. They formulated the problem as a multi-level capacitated facility location problem on a tree topology with nonlinear link cost. The major factor of classification of facility location-allocation problems is associated with the objective function. In the objective function, when the maximum distance between a demand node and the nearest facility is minimized, the problem is categorized as the p-center problem (Albareda-Sambola et al., 2010; Kariv \& Hakimi, 1979). There are various types of this problem: the "absolute" $p$-center problem permits the facilities to be anywhere along the arcs while the "vertex" $p$-center problem restricts the set of candidate facility sites to the nodes of the network (Revelle et al., 2008). Both types can be either weighted or unweighted. In the unweighted problem, all 
demand nodes are treated equally, and in the weighted model, the distances between demand nodes and facilities are multiplied by a weight associated with the demand node (Lu \& Sheu, 2013). In the realworld cases, the exact values of some parameters of facility location problems are unavailable and have uncertain properties. Therefore, the assumption of uncertainty is needed for parameters of problem and to deal with this uncertainty, different methods like stochastic programming and possibility theory have been developed.

In stochastic facility location problems, the uncertain parameters are distinguished by random variables where their probability distribution is available. Schutz et al. (2008) formulated the facility location problem as a two-stage stochastic programming method and used a solution method based on Lagrangian relaxation. Xu et al. (2013) considered a primal-dual 3-approximation algorithm for the stochastic facility location problem with submodular penalties. Döyen et al. (2012) developed a twostage stochastic programming model for a humanitarian relief logistics problem where the objective was to minimize the total cost of facility location, inventory holding, transportation and shortage. Based on possibility theory and fuzzy set theory, which deals with ambiguous and imprecise concepts, other methods have been developed for solving the facility location problems with uncertain parameters. For instance, Küçükdeniz et al. (2012) studied a fuzzy c-means clustering algorithm based method for solving a capacitated multi-facility location problem, which is involved the integrated use of fuzzy cmeans and convex programming. Wang et al. (2009) proposed a two-stage fuzzy facility location problem with value-at-risk and represented the fuzzy parameters of the location problem in the form of continuous fuzzy variables. Recently, a two-stage capacitated facility location model with fuzzy costs and demands has been developed by Wang and Watada (2013). Furthermore, Ishii et al. (2007) proposed fuzzy facility location problem with preference of candidate sites.

In the real-world situation, the parameters of a facility location problem can embrace fuzziness and randomness at the same time. In other words, available random data of parameters of the problem may be unsatisfactory; therefore, the fuzzy information must be integrated with the available random data. Indeed, fuzziness and randomness of parameters are mixed up with each other and defined a hybrid uncertain variable in the facility location problem. Wen and Kang (2011) presented optimal models for the facility location problem with random fuzzy demands and solved the problem through the simplex algorithm, random fuzzy simulations and a genetic algorithm. Wang and Watada (2012) studied a hybrid modified PSO approach to VaR-based facility location problems with variable capacity in the fuzzy random uncertainty. This paper proposes the vertex $p$-center problem with uncertain demandweighted distance in which the demands are considered as fuzzy random variables (FRVs). The most important aim of this paper is to introduce new approaches for the problem, by using possibility and necessity measures which are based on the possibility theory (Dubois \& Prade, 2001) and fuzzy random chance-constrained programming (FRCCP). Therefore, the theorems are obtained to convert the original problem to the deterministic integer programming (IP) problem for optimistic and pessimistic decision makers (DMs) separately and simultaneously.

The remainder of this paper is organized as follows. In section 2, a vertex $p$-center problem with fuzzy random demand-weighted distance is introduced. In section 3, the possibility theory and FRCCP are implemented to convert the problem to the deterministic IP problem. Section 4 illustrates the real case study of bicycle stations in the city of Tabriz in Iran and computational results are reported to clarify the methods described in this paper. Finally, conclusions and discussions of future research are given in Section 5 .

\section{Problem formulation}

The $p$-center problem as a facility location problem, also known as the minimax problem, is a wellknown problem. This problem minimizes the coverage distance with a giving number of facilities, while maintaining the coverage of all demand nodes. In this problem, the objective is to find locations of $p$ facilities so that all demands are covered and the maximum distance between a demand node and 
the nearest facility is minimized (Farahani et al., 2010). In this section, mathematical programming models of vertex $p$-center problem with demand-weighted distance will be introduced.

\subsection{Vertex P-center Problem with Demand-Weighted Distance}

In some cases, demand-weighted distance is considered by Daskin (2011) in vertex $p$-center problem formulated by Hakimi (1965). The assumptions of this problem are collected as: (a) there are $P$ facilities to be located, (b) The facilities will be located on the nodes of the network, (c) The capacities of facilities are unlimited, (d) Demand points are on the nodes of network, and (e) Demand nodes are weighted (Farahani et al., 2010).

The IP formulation of the vertex $p$-center problem with demand-weighted distance is given as follows:

$\min Z$

subject to

$\sum_{j} X_{i j}=1 ; \quad \forall i$

$\sum_{j} Y_{j}=p$

$X_{i j} \leq Y_{j}$,

$Z \geq h_{i} \sum_{j} d_{i j} X_{i j} ; \quad \forall i$,

$X_{i j}, Y_{j} \in\{0,1\} ; \quad \forall i, j$,

where

$i=$ set of demand nodes,

$j=$ set of candidate facility sites,

$d_{i j}=$ length of the shortest path between demand node $i$ and candidate facility site $j$,

$p=$ number of facilities to be located,

$Y_{j}= \begin{cases}1 & \text { if a facility is located at candidate site } j, \\ 0 & \text { otherwise }\end{cases}$

$X_{i j}= \begin{cases}1 & \text { if demand node } i \text { is assigned to an open facility at candidate site } j, \\ 0 & \text { otherwise }\end{cases}$

$h_{i}=$ demand at node $i$,

$Z$ = maximum distance between a demand node and the nearest facility.

The objective function (1) minimizes the maximum distance between each demand node and its closest open facility. Constraint (2) states that the demand at node $i$ must be assigned to a facility at some node $j$ for all nodes $i$. Constraint (3) guarantees that $p$ facilities are located. Constraint (4) ensures that assignments can only be made to open facilities. Constraints (5) define the maximum distance between any demand node $i$ and the nearest facility at node $j$. Finally, constraints (6) refer to integrality constraints. The inputs of this model are $d_{i j}, p, h_{i}$ and the outputs are $X_{i j}, Y_{j}, Z$.

\subsection{Vertex P-center Problem with Uncertain Demand-Weighted Distance}

In the real-world case, the exact values of demands are unavailable and have uncertain properties. In this paper, vertex $p$-center problem is considered with uncertain demand-weighted distance by using 
the concepts of FRVs. Fuzziness and randomness are two types of common uncertainties in problems and the concept of FRVs is introduced as an analogous notion to random variables in order to extend statistical analysis to situations when the outcomes of some random experiment are fuzzy sets. A FRV is one of the proper ways to describe this type of uncertainty. It was first introduced by Kwakernaak (1978) and then developed by Puri and Ralescu (1986). In general, FRVs can be defined in a $n$ dimensional Euclidian space $R^{n}$. In this paper, the definition of FRVs will be presented in a single dimensional Euclidian space $R$.

\section{Definition 1:}

Let $(\Omega, \mathrm{A}, \mathrm{P})$ be a probability space, where $\Omega$ is a sample space, $\mathrm{A}$ is a $\sigma$-field and $\mathrm{P}$ is a probability measure. Let $F_{N}$ be the set of all fuzzy numbers and B a Borel $\sigma$-field of R. Then a map $\tilde{Z}: \Omega \rightarrow F_{N}$ is called a FRV if it holds that

$$
\left\{(\omega, \tau) \in \Omega \times R \mid \tau \in \tilde{\bar{Z}}_{\alpha}(\omega)\right\} \in A \times B, \forall \alpha \in[0,1]
$$

where

$$
\tilde{Z}_{\alpha}(\omega)=\left[\tilde{\bar{Z}}_{\alpha}^{-}(\omega), \tilde{\bar{Z}}_{\alpha}^{+}(\omega)\right]=\left\{\tau \in R \mid \mu_{\bar{z}(\omega)}(\tau) \geq \alpha\right\}
$$

is an $\alpha$-level set of the fuzzy number $\tilde{Z}(\omega)$ for $\omega \in \Omega$.

Intuitively, FRVs are considered as random variables whose actual values are not real values but fuzzy numbers or fuzzy sets.

\section{Definition 2:}

LR fuzzy number $\tilde{A}$ is defined by the following membership function:

$$
\tilde{A}(X)=\left\{\begin{array}{lll}
L\left(\frac{A^{0}-x}{\beta}\right) & \text { if } & A^{0}-\beta \leq x<A^{0} \\
1 & \text { if } & A^{0} \leq x \leq A^{1} \\
R\left(\frac{x-A^{1}}{\gamma}\right) & \text { if } & A^{1}<x \leq A^{1}+\gamma
\end{array}\right.
$$

where $\left[A^{0}, A^{1}\right]$ shows the peak of fuzzy number $\tilde{A}$ and $\beta, \gamma$ represent the left and right spread respectively; $L, R=[0,1] \rightarrow[0,1]$ with $L(0)=R(0)=1$ and $L(1)=R(1)=0$ are strictly decreasing, continuous functions. A possible representation of a LR fuzzy number is $\tilde{A}=\left(A^{0}, A^{1}, \beta, \gamma\right)_{L R}$. In order to consider the vertex $p$-center problem with uncertain demand-weighted distance, the concept of FRVs will be implemented. In the following problem, called vertex $p$-center problem with fuzzy random demandweighted distance, the demand at node $i\left(h_{i}\right)$ is considered as a FRV:

\section{Problem 1}

$\min Z$

subject to

$$
\begin{aligned}
& \sum_{j} X_{i j}=1 ; \quad \forall i, \\
& \sum_{j} Y_{j}=p, \\
& X_{i j} \leq Y_{j}, \\
& Z \geq \tilde{\bar{h}}_{i} \sum_{j} d_{i j} X_{i j} ; \quad \forall i,
\end{aligned}
$$


$X_{i j}, Y_{j} \in\{0,1\} ; \quad \forall i, j$,

where $\tilde{\overline{h_{i}}}=\left(\bar{h}_{i}^{0}, \bar{h}_{i}^{1}, \beta_{i}, \gamma_{i}\right)_{L R}$ represents a FRV whose observed value for each $\omega \in \Omega$ is fuzzy number $\tilde{h}_{i}(\omega)=\left(h_{i}^{0}(\omega), h_{i}^{1}(\omega), \beta_{i}, \gamma_{i}\right)_{L R}$. Furthermore, $\left(\bar{h}_{i}^{0}, \bar{h}_{i}^{1}\right)=\left(h_{i}^{(0)}+\bar{t} h_{i}^{(2)}, h_{i}^{(1)}+\bar{t} h_{i}^{(2)}\right)$ is a random vector in which $\bar{t}$ is a random variable with cumulative distribution function $T$.

\section{Methodology}

In order to transform the fuzzy random programming to the deterministic IP model, the possibility and necessity measures will be implemented to the constraint (14). The degree of possibility and necessity will be defined to the constraint whose elements are FRVs.

\subsection{Possibility-based Model}

The possibility degree of constraint $Z \geq \tilde{h}_{i}(\omega) \sum_{j} d_{i j} X_{i j}$ is defined as follows:

$\operatorname{Pos}\left(Z \geq \tilde{h}_{i}(\omega) \sum_{j} d_{i j} X_{i j}\right)=\sup _{y_{1}, y_{2}}\left\{\min \left\{\mu_{Z}\left(y_{1}\right), \mu_{\tilde{h}_{i}(\omega)} \sum_{j} d_{i j} X_{i j}\left(y_{2}\right)\right\} \mid y_{1} \geq y_{2}\right\}$

FRVs in the constraint (14) of problem 1 will be handled through FRCCP by the following problem:

Problem 2

$\min Z$

subject to

$\sum_{j} X_{i j}=1 ; \quad \forall i$

$\sum_{j} Y_{j}=p$,

$X_{i j} \leq Y_{j}$,

$\operatorname{Pr}\left\{\omega \mid \operatorname{Pos}\left(Z \geq \tilde{h}_{i}(\omega) \sum_{j} d_{i j} X_{i j}\right) \geq \eta\right\} \geq \lambda ; \quad \forall i$,

$X_{i j}, Y_{j} \in\{0,1\} ; \quad \forall i, j$,

where $\lambda$ and $\eta$ are predetermined probability and possibility levels, respectively. The optimal solution of this problem is called possibility optimal solution of the vertex $p$-center problem with fuzzy random demand-weighted distance. To transform this problem to the deterministic IP, constraint (20) should be reformulated through the following theorem:

Theorem1:

$\operatorname{Pr}\left\{\omega \mid \operatorname{Pos}\left(Z \geq \tilde{h}_{i}(\omega) \sum_{j} d_{i j} X_{i j}\right) \geq \eta\right\} \geq \lambda \Leftrightarrow Z \geq\left(h_{i}^{(0)}+T^{*}(\lambda) h_{i}^{(2)}-\beta_{i} L^{*}(\eta)\right) \sum_{j} d_{i j} X_{i j}$

where $T^{*}$ and $L^{*}$ are pseudo inverse function defined as $T^{*}(\lambda)=\inf \{t \mid T(t) \geq \lambda\}, L^{*}(\lambda)=\sup \{t \mid L(t) \geq \lambda\}$.

The proof of theorem 1 is available in Appendix A. Based on theorem 1, through FRCCP, the problem 2 is converted to the following deterministic IP problem:

Problem 3

$\min Z$

subject to 
$\sum_{j} X_{i j}=1 ; \quad \forall i$,

$\sum_{j} Y_{j}=p$,

$X_{i j} \leq Y_{j}$,

$Z \geq\left(h_{i}^{(0)}+T^{*}(\lambda) h_{i}^{(2)}-\beta_{i} L^{*}(\eta)\right) \sum_{j} d_{i j} X_{i j} ; \quad \forall i$,

$X_{i j}, Y_{j} \in\{0,1\} ; \quad \forall i, j$.

Consequently, the possibility optimal solution of original problem 1 is equal to the optimal solution of problem 3.

\subsection{Necessity-based Model}

In the previous section, the possibility degree has been considered for the vertex $p$-center problem with fuzzy random demand-weighted distance, which is useful in making a decision with an optimistic notion. Possibility-based model may be improper since the obtained solution will be too optimistic. Therefore, a necessity-based model can be suitable for pessimistic DM, so this section devotes to investigate the problem by using necessity degrees.

The necessity degree of constraint $Z \geq \tilde{h}_{i}(\omega) \sum_{j} d_{i j} X_{i j}$ is defined as follows:

$\operatorname{Nec}\left(Z \geq \tilde{h}_{i}(\omega) \sum_{j} d_{i j} X_{i j}\right)=\inf _{y_{1}, y_{2}}\left\{\max \left\{1-\mu_{Z}\left(y_{1}\right), 1-\mu_{\tilde{h}_{i}(\omega)} \sum_{j} d_{i j} X_{i j}\left(y_{2}\right)\right\} \mid y_{1} \geq y_{2}\right\}$.

Like the possibility-based model, FRVs in the constraint (14) of problem 1 will be handled through FRCCP by the following problem:

Problem 4

$\min Z$

subject to

$\sum_{j} X_{i j}=1 ; \quad \forall i$,

$\sum_{j} Y_{j}=p$,

$X_{i j} \leq Y_{j}$,

$\operatorname{Pr}\left\{\omega \mid \operatorname{Nec}\left(Z \geq \tilde{h}_{i}(\omega) \sum_{j} d_{i j} X_{i j}\right) \geq \eta\right\} \geq \lambda ; \quad \forall i$,

$X_{i j}, Y_{j} \in\{0,1\} ; \quad \forall i, j$.

Like the previous section, $\lambda$ and $\eta$ are predetermined probability and possibility levels, respectively. The optimal solution of this problem is called necessity optimum solution of the vertex $p$-center problem with fuzzy random demand-weighted distance. To transform this problem to the deterministic IP, constraint (32) should be reformulated through the following theorem:

Theorem2:

$\operatorname{Pr}\left\{\omega \mid \operatorname{Nec}\left(Z \geq \tilde{h}_{i}(\omega) \sum_{j} d_{i j} X_{i j}\right) \geq \eta\right\} \geq \lambda \Leftrightarrow Z \geq\left(h_{i}^{(0)}+T^{*}(\lambda) h_{i}^{(2)}-\beta_{i} L^{*}(1-\eta)\right) \sum_{j} d_{i j} X_{i j}$.

The proof of theorem 2 is available in Appendix B. Consequently, the necessity optimal solution of problem 1 is derived by the following problem: 
Problem 5

$\min Z$

subject to

$\sum_{j} X_{i j}=1 ; \quad \forall i$

$\sum_{j} Y_{j}=p$,

$X_{i j} \leq Y_{j}$,

$Z \geq\left(h_{i}^{(0)}+T^{*}(\lambda) h_{i}^{(2)}-\beta_{i} L^{*}(1-\eta)\right) \sum_{j} d_{i j} X_{i j} ; \quad \forall i$,

$X_{i j}, Y_{j} \in\{0,1\} ; \quad \forall i, j$.

\subsection{Hybrid-based model}

Possibility and necessity-based models can satisfy the optimistic and pessimistic DMs separately. Now, the aim of this section is to introduce a model, which satisfies both optimistic and pessimistic DMs as a hybrid-based model. In the hybrid-based model, the possibility and necessity measures are applied with together and by using the results of theorems 1 and 2, the model is written as follows:

Problem 6

$\min Z$

subject to

$\sum_{j} X_{i j}=1 ; \quad \forall i$

$\sum_{j} Y_{j}=p$,

$X_{i j} \leq Y_{j}$,

$Z \geq\left(h_{i}^{(0)}+T^{*}(\lambda) h_{i}^{(2)}-\beta_{i} L^{*}(\eta)\right) \sum_{j} d_{i j} X_{i j} ; \quad \forall i$,

$Z \geq\left(h_{i}^{(0)}+T^{*}(\lambda) h_{i}^{(2)}-\beta_{i} L^{*}(1-\eta)\right) \sum_{j} d_{i j} X_{i j} ; \quad \forall i$,

$X_{i j}, Y_{j} \in\{0,1\} ; \quad \forall i, j$.

All obtained problems, which are deterministic IP can be solved by mixed integer programming solvers.

\section{A real case study of bicycle stations in Tabriz city}

To emphasize the implementation of this study for a real-world case study, a case study of location of the bicycle stations in Tabriz city, an urban area in the north-western of Iran, has been considered. The government has decided to allocate the bicycle stations in this area and because of the budget limitation, ten stations will be considered.

Fig. 1 shows the map of Tabriz city in which the stations will be located. With dispersed population in this area, it is essential to design a method to provide a good solution with a low number of stations. Therefore, for providing this solution, the vertex $p$-center problem with uncertain demand-weighted distance was proposed. This case study contains fifteen demand nodes illustrated in Fig. 2. The shortest distances between each pair of nodes are collected in Table 1 as a distance matrix. 


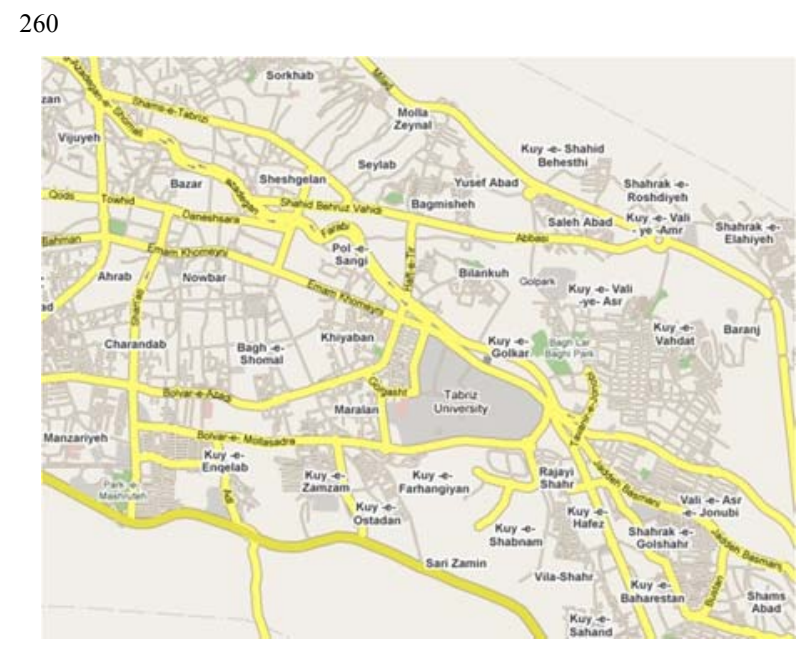

Fig. 1. Map of Tabriz city

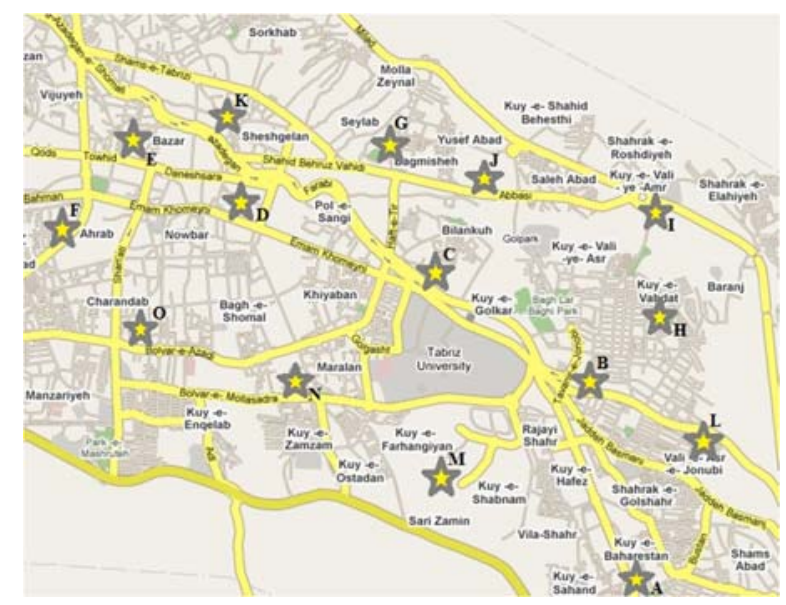

Fig. 2. Location of fifteen demand nodes

Table 1

The shortest distance between each pair of nodes (in Km)

\begin{tabular}{|c|c|c|c|c|c|c|c|c|c|c|c|c|c|c|c|}
\hline & A & B & $\mathrm{C}$ & $\mathrm{D}$ & $\mathrm{E}$ & $\mathrm{F}$ & $\mathrm{G}$ & $\mathrm{H}$ & $\mathrm{I}$ & $\mathrm{J}$ & $\mathrm{K}$ & $\mathrm{L}$ & $\mathrm{M}$ & $\mathrm{N}$ & $\mathrm{O}$ \\
\hline $\mathrm{A}$ & - & 3.8 & 5.9 & 8.6 & 9.1 & 9.8 & 8 & 4.8 & 6.9 & 6.8 & 10.5 & 3.7 & 3.9 & 6.1 & 8 \\
\hline B & - & - & 2.1 & 4.8 & 5.8 & 6 & 4 & 2 & 4.1 & 3.8 & 6.1 & 1 & 1 & 4 & 6.4 \\
\hline $\mathrm{C}$ & - & - & - & 2.7 & 3.7 & 3.9 & 1.9 & 3.4 & 4.9 & 3.5 & 3.8 & 3.5 & 3.1 & 2.7 & 3.5 \\
\hline D & - & - & - & - & 1 & 1.3 & 2.9 & 6.9 & 6.3 & 5 & 1.4 & 6.1 & 5.8 & 2.8 & 2.1 \\
\hline E & - & - & - & - & - & 1 & 3 & 7.6 & 6.5 & 4.8 & 1.6 & 7.1 & 6.7 & 3.8 & 2.6 \\
\hline $\mathrm{F}$ & - & - & - & - & - & - & 3.9 & 7.9 & 7.4 & 5.7 & 2.5 & 7.4 & 7 & 4 & 2.5 \\
\hline $\mathrm{G}$ & - & - & - & - & - & - & - & 4.5 & 3.2 & 1.7 & 1.6 & 6.7 & 5 & 3.7 & 4.7 \\
\hline $\mathrm{H}$ & - & - & - & - & - & - & - & - & 2 & 2.9 & 6.1 & 1.3 & 3 & 5.5 & 7 \\
\hline I & - & - & - & - & - & - & - & - & - & 1.6 & 4.8 & 3.2 & 4.8 & 6.9 & 7.7 \\
\hline $\mathrm{J}$ & - & - & - & - & - & - & - & - & - & - & 3.2 & 3.9 & 5.4 & 5.2 & 5.6 \\
\hline $\mathrm{K}$ & - & - & - & - & - & - & - & - & - & - & - & 7.3 & 7.2 & 3.5 & 3.2 \\
\hline $\mathrm{L}$ & - & - & - & - & - & - & - & - & - & - & - & - & 2.5 & 5 & 6.7 \\
\hline $\mathrm{M}$ & - & - & - & - & - & - & - & - & - & - & - & - & - & 3.2 & 5 \\
\hline $\mathrm{N}$ & - & - & - & - & - & - & - & - & - & - & - & - & - & - & 1.8 \\
\hline $\mathrm{O}$ & - & - & - & - & - & - & - & - & - & - & - & - & - & - & - \\
\hline
\end{tabular}

Because of the dispersed population of this area and uncertainty about demands, in the demandweighted distance model, demand nodes were considered as FRVs. Let $\tilde{\bar{h}}_{i}=\left(\bar{h}_{i}^{0}, \bar{h}_{i}^{1}, \beta_{i}, \gamma_{i}\right)_{L R}$ be a FRV where $\bar{h}_{i}^{0}, \bar{h}_{i}^{1}$ are random variables defined as $\bar{h}_{i}^{0}=h_{i}^{(0)}+\bar{t} h_{i}^{(2)}$ and $\bar{h}_{i}^{1}=h_{i}^{(1)}+\bar{t} h_{i}^{(2)}$. For each node, values of these FRVs have been collected through a survey and support of municipality of Tabriz city and Table 2 illustrates these values with 5 -dimentional vectors $\left(h_{i}^{(0)}, h_{i}^{(1)}, h_{i}^{(2)}, \beta_{i}, \gamma_{i}\right)$.

Table 2

Values of fuzzy random variables for each node

\begin{tabular}{cccccc}
\hline$i$ & \multicolumn{7}{c}{$\left(h_{i}^{(0)}, h_{i}^{(1)}, h_{i}^{(2)}, \beta_{i}, \gamma_{i}\right)$} \\
\hline \multicolumn{7}{c}{} & $(80,100,3,9,10)$ & $\mathrm{F}$ & $(45,50,5,4,5)$ & $\mathrm{K}$ & $(40,45,4,4,5)$ \\
$\mathrm{A}$ & $(40,45,5,5,4)$ & $\mathrm{G}$ & $(20,25,6,2,3)$ & $\mathrm{L}$ & $(50,55,4,6,5)$ \\
$\mathrm{B}$ & $(50,65,4,4,5)$ & $\mathrm{H}$ & $(37,43,5,3,4)$ & $\mathrm{M}$ & $(20,35,5,2,3)$ \\
$\mathrm{C}$ & $(60,70,4,6,4)$ & $\mathrm{I}$ & $(28,35,5,3,3)$ & $\mathrm{N}$ & $(15,20,6,3,2)$ \\
$\mathrm{D}$ & $(45,50,6,5,4)$ & $\mathrm{J}$ & $(25,30,5,3,2)$ & $\mathrm{O}$ & $(30,35,5,3,4)$ \\
$\mathrm{E}$ & & &
\end{tabular}

Now, by applying the possibility, necessity and hybrid-based models to the vertex $p$-center problem with uncertain demand-weighted distance, the possibility, necessity and hybrid optimal solutions will be obtained for different levels of probability and possibility $\{0.1,0.3,0.5,0.7,0.9\}$. The optimal solutions of all models are collected in the parts (a), (b) and (c) of Table 3, which shows the objective function value of the problem and also the optimal locations of the bicycle stations. 
Table 3

Numerical results of location of the bicycle stations

\begin{tabular}{|c|c|c|c|c|c|}
\hline \multicolumn{6}{|c|}{ Part a: Possibility optimal solution } \\
\hline & & & $\eta$ & & \\
\hline$\lambda$ & 0.1 & 0.3 & 0.5 & 0.7 & 0.9 \\
\hline 0.1 & $\begin{array}{c}32.76^{\mathrm{OFV}} \\
(\mathrm{A}, \mathrm{C}, \mathrm{D}, \mathrm{F}, \mathrm{H}, \mathrm{I}, \mathrm{K}, \mathrm{L}, \mathrm{M}, \mathrm{O})^{\mathrm{OLBS}}\end{array}$ & $\begin{array}{c}33.76 \\
\text { (A.C.DF.H.J.L.M.O) }\end{array}$ & 34.76 & $\begin{array}{c}35.76 \\
\mathrm{~B} C \mathrm{~K}\end{array}$ & 36.76 \\
\hline 0.3 & $\begin{array}{c}37.38 \\
(\mathrm{~A} B \mathrm{CDF} \text {. }\end{array}$ & $\begin{array}{c}38.38 \\
\text { (ABCDEHJKLO) }\end{array}$ & $\begin{array}{c}39.38 \\
39 . \mathrm{A}, \mathrm{L}, \mathrm{L})\end{array}$ & $\begin{array}{c}40.38 \\
4, \mathrm{~N}, \mathrm{~L}, \mathrm{~L}, \mathrm{~N})\end{array}$ & 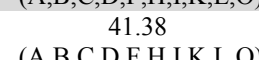 \\
\hline 0.5 & $\begin{array}{c}(\mathrm{A}, \mathrm{B}, \mathrm{C}, \mathrm{L}, \mathrm{F}, \mathrm{H}, \mathrm{I}, \mathrm{K}, \mathrm{L}, \mathrm{O}) \\
(\mathrm{A}, \mathrm{C}, \mathrm{D}, \mathrm{F}, \mathrm{H}, \mathrm{I}, \mathrm{K}, \mathrm{L}, \mathrm{M}, \mathrm{O})\end{array}$ & $\begin{array}{c}(\mathrm{A}, \mathrm{B}, \mathrm{C}, \mathrm{L}, \mathrm{F}, \mathrm{H}, \mathrm{I}, \mathrm{K}, \mathrm{L}, \mathrm{O}) \\
(\mathrm{A}, \mathrm{C}, \mathrm{D}, \mathrm{F}, \mathrm{H}, \mathrm{I}, \mathrm{K}, \mathrm{L}, \mathrm{M}, \mathrm{O})\end{array}$ & $\begin{array}{c}(\mathrm{A}, \mathrm{B}, \mathrm{C}, \mathrm{D}, \mathrm{F}, \mathrm{H}, \mathrm{L}, \mathrm{K}, \mathrm{L}, \mathrm{U}) \\
(\mathrm{A}, \mathrm{C}, \mathrm{D}, \mathrm{F}, \mathrm{H}, \mathrm{I}, \mathrm{K}, \mathrm{L}, \mathrm{M}, \mathrm{O})\end{array}$ & $\begin{array}{c}(\mathrm{A}, \mathrm{B}, \mathrm{C}, \mathrm{D}, \mathrm{F}, \mathrm{H}, \mathrm{l}, \mathrm{K}, \mathrm{L}, \mathrm{U}) \\
43.50 \\
(\mathrm{~A}, \mathrm{C}, \mathrm{D}, \mathrm{F}, \mathrm{H}, \mathrm{I}, \mathrm{K}, \mathrm{L}, \mathrm{M}, \mathrm{O})\end{array}$ & $\begin{array}{c}(\mathrm{A}, \mathrm{B}, \mathrm{C}, \mathrm{D}, \mathrm{H}, \mathrm{H}, \mathrm{l}, \mathrm{K}, \mathrm{L}, \mathrm{O} \\
(\mathrm{A}, \mathrm{C}, \mathrm{D}, \mathrm{F}, \mathrm{H}, \mathrm{I}, \mathrm{K}, \mathrm{L}, \mathrm{M}, \mathrm{O})\end{array}$ \\
\hline 0.7 & $\begin{array}{c}43.62 \\
(\mathrm{~A}, \mathrm{C}, \mathrm{D}, \mathrm{F}, \mathrm{H}, \mathrm{I}, \mathrm{K}, \mathrm{L}, \mathrm{M}, \mathrm{O})\end{array}$ & $\begin{array}{c}44.62 \\
(\mathrm{~A}, \mathrm{C}, \mathrm{D}, \mathrm{F}, \mathrm{H}, \mathrm{I}, \mathrm{K}, \mathrm{L}, \mathrm{M}, \mathrm{O})\end{array}$ & $\begin{array}{c}45.60 \\
(\mathrm{~A}, \mathrm{C}, \mathrm{D}, \mathrm{E}, \mathrm{H}, \mathrm{I}, \mathrm{K}, \mathrm{L}, \mathrm{M}, \mathrm{O})\end{array}$ & $\begin{array}{c}46.40 \\
(\mathrm{~A}, \mathrm{C}, \mathrm{D}, \mathrm{E}, \mathrm{H}, \mathrm{I}, \mathrm{K}, \mathrm{L}, \mathrm{M}, \mathrm{O})\end{array}$ & $\begin{array}{c}47.20 \\
(\mathrm{~A}, \mathrm{C}, \mathrm{D}, \mathrm{E}, \mathrm{H}, \mathrm{I}, \mathrm{K}, \mathrm{L}, \mathrm{M}, \mathrm{O})\end{array}$ \\
\hline 0.9 & $\begin{array}{c}47.85 \\
(\mathrm{~A}, \mathrm{C}, \mathrm{D}, \mathrm{E}, \mathrm{H}, \mathrm{I}, \mathrm{K}, \mathrm{L}, \mathrm{M}, \mathrm{O})\end{array}$ & $\begin{array}{c}48.65 \\
(\mathrm{~A}, \mathrm{C}, \mathrm{D}, \mathrm{E}, \mathrm{H}, \mathrm{I}, \mathrm{K}, \mathrm{L}, \mathrm{M}, \mathrm{O})\end{array}$ & $\begin{array}{c}49.45 \\
(\mathrm{~A}, \mathrm{~B}, \mathrm{C}, \mathrm{D}, \mathrm{E}, \mathrm{H}, \mathrm{I}, \mathrm{K}, \mathrm{L}, \mathrm{O})\end{array}$ & $\begin{array}{c}50.25 \\
(\mathrm{~A}, \mathrm{~B}, \mathrm{C}, \mathrm{D}, \mathrm{E}, \mathrm{H}, \mathrm{I}, \mathrm{K}, \mathrm{L}, \mathrm{O})\end{array}$ & $\begin{array}{c}51.05 \\
(\mathrm{~A}, \mathrm{~B}, \mathrm{C}, \mathrm{D}, \mathrm{E}, \mathrm{H}, \mathrm{I}, \mathrm{K}, \mathrm{L}, \mathrm{O})\end{array}$ \\
\hline
\end{tabular}

OFV: Objective Function Value, OLBS: Optimal Locations of the Bicycle Stations

\begin{tabular}{cccccc}
\hline \multicolumn{5}{c}{ Part b: Necessity optimal solution } \\
\hline \multicolumn{7}{c}{0.1} & 0.3 & 0.5 & 0.9 \\
\hline \multirow{2}{*}{0.1} & 36.76 & 35.76 & 34.76 & 0.7 & 32.76 \\
& $(\mathrm{~A}, \mathrm{~B}, \mathrm{C}, \mathrm{D}, \mathrm{F}, \mathrm{H}, \mathrm{I}, \mathrm{K}, \mathrm{L}, \mathrm{O})$ & $(\mathrm{A}, \mathrm{B}, \mathrm{C}, \mathrm{D}, \mathrm{F}, \mathrm{H}, \mathrm{I}, \mathrm{K}, \mathrm{L}, \mathrm{O})$ & $(\mathrm{A}, \mathrm{B}, \mathrm{C}, \mathrm{D}, \mathrm{F}, \mathrm{H}, \mathrm{I}, \mathrm{K}, \mathrm{L}, \mathrm{O})$ & $(\mathrm{A}, \mathrm{C}, \mathrm{D}, \mathrm{F}, \mathrm{H}, \mathrm{I}, \mathrm{K}, \mathrm{L}, \mathrm{M}, \mathrm{O})$ & $(\mathrm{A}, \mathrm{C}, \mathrm{D}, \mathrm{F}, \mathrm{H}, \mathrm{I}, \mathrm{K}, \mathrm{L}, \mathrm{M}, \mathrm{O})$ \\
0.3 & 41.38 & 40.38 & 39.38 & 37.38 \\
& $(\mathrm{~A}, \mathrm{~B}, \mathrm{C}, \mathrm{D}, \mathrm{F}, \mathrm{H}, \mathrm{I}, \mathrm{K}, \mathrm{L}, \mathrm{O})$ & $(\mathrm{A}, \mathrm{B}, \mathrm{C}, \mathrm{D}, \mathrm{F}, \mathrm{H}, \mathrm{I}, \mathrm{K}, \mathrm{L}, \mathrm{O})$ & $(\mathrm{A}, \mathrm{B}, \mathrm{C}, \mathrm{D}, \mathrm{F}, \mathrm{H}, \mathrm{I}, \mathrm{K}, \mathrm{L}, \mathrm{O})$ & $(\mathrm{A}, \mathrm{B}, \mathrm{C}, \mathrm{D}, \mathrm{F}, \mathrm{H}, \mathrm{I}, \mathrm{K}, \mathrm{L}, \mathrm{O})$ & $(\mathrm{A}, \mathrm{B}, \mathrm{C}, \mathrm{D}, \mathrm{F}, \mathrm{H}, \mathrm{I}, \mathrm{K}, \mathrm{L}, \mathrm{O})$ \\
0.5 & 44.50 & 42.50 & 40.50 \\
& $(\mathrm{~A}, \mathrm{C}, \mathrm{D}, \mathrm{F}, \mathrm{H}, \mathrm{I}, \mathrm{K}, \mathrm{L}, \mathrm{M}, \mathrm{O})$ & $(\mathrm{A}, \mathrm{C}, \mathrm{D}, \mathrm{F}, \mathrm{H}, \mathrm{I}, \mathrm{K}, \mathrm{L}, \mathrm{M}, \mathrm{O})$ & $(\mathrm{A}, \mathrm{C}, \mathrm{D}, \mathrm{F}, \mathrm{H}, \mathrm{I}, \mathrm{K}, \mathrm{L}, \mathrm{M}, \mathrm{O})$ & $(\mathrm{A}, \mathrm{C}, \mathrm{D}, \mathrm{F}, \mathrm{H}, \mathrm{I}, \mathrm{K}, \mathrm{L}, \mathrm{M}, \mathrm{O})$ & $(\mathrm{A}, \mathrm{C}, \mathrm{D}, \mathrm{F}, \mathrm{H}, \mathrm{I}, \mathrm{K}, \mathrm{L}, \mathrm{M}, \mathrm{O})$ \\
0.7 & 47.20 & 46.40 & 45.60 & 43.62 \\
& $(\mathrm{~A}, \mathrm{C}, \mathrm{D}, \mathrm{E}, \mathrm{H}, \mathrm{I}, \mathrm{K}, \mathrm{L}, \mathrm{M}, \mathrm{O})$ & $(\mathrm{A}, \mathrm{C}, \mathrm{D}, \mathrm{E}, \mathrm{H}, \mathrm{I}, \mathrm{K}, \mathrm{L}, \mathrm{M}, \mathrm{O})$ & $(\mathrm{A}, \mathrm{C}, \mathrm{D}, \mathrm{E}, \mathrm{H}, \mathrm{I}, \mathrm{K}, \mathrm{L}, \mathrm{M}, \mathrm{O})$ & $(\mathrm{A}, \mathrm{C}, \mathrm{D}, \mathrm{F}, \mathrm{H}, \mathrm{I}, \mathrm{K}, \mathrm{L}, \mathrm{M}, \mathrm{O})$ & $(\mathrm{A}, \mathrm{C}, \mathrm{D}, \mathrm{F}, \mathrm{H}, \mathrm{I}, \mathrm{K}, \mathrm{L}, \mathrm{M}, \mathrm{O})$ \\
0.9 & 51.05 & 50.25 & 49,45 & 47.85 \\
& $(\mathrm{~A}, \mathrm{~B}, \mathrm{C}, \mathrm{D}, \mathrm{E}, \mathrm{H}, \mathrm{I}, \mathrm{K}, \mathrm{L}, \mathrm{O})$ & $(\mathrm{A}, \mathrm{B}, \mathrm{C}, \mathrm{D}, \mathrm{E}, \mathrm{H}, \mathrm{I}, \mathrm{K}, \mathrm{L}, \mathrm{O})$ & $(\mathrm{A}, \mathrm{B}, \mathrm{C}, \mathrm{D}, \mathrm{E}, \mathrm{H}, \mathrm{I}, \mathrm{K}, \mathrm{L}, \mathrm{O})$ & $(\mathrm{A}, \mathrm{C}, \mathrm{D}, \mathrm{E}, \mathrm{H}, \mathrm{I}, \mathrm{K}, \mathrm{L}, \mathrm{M}, \mathrm{O})$ & $(\mathrm{A}, \mathrm{C}, \mathrm{D}, \mathrm{E}, \mathrm{H}, \mathrm{I}, \mathrm{K}, \mathrm{L}, \mathrm{M}, \mathrm{O})$ \\
\hline
\end{tabular}

\begin{tabular}{|c|c|c|c|c|c|}
\hline \multirow[b]{3}{*}{$\lambda$} & \multicolumn{5}{|c|}{ Part c: Hybrid optimal solution } \\
\hline & & & $\eta$ & & \\
\hline & 0.1 & 0.3 & 0.5 & 0.7 & 0.9 \\
\hline \multirow[b]{2}{*}{0.1} & 36.76 & 35.76 & 34.76 & 35.76 & 36.76 \\
\hline & $(\mathrm{A}, \mathrm{B}, \mathrm{C}, \mathrm{D}, \mathrm{F}, \mathrm{H}, \mathrm{I}, \mathrm{K}, \mathrm{L}, \mathrm{O})$ & $(\mathrm{A}, \mathrm{B}, \mathrm{C}, \mathrm{D}, \mathrm{F}, \mathrm{H}, \mathrm{I}, \mathrm{K}, \mathrm{L}, \mathrm{O})$ & $(\mathrm{A}, \mathrm{B}, \mathrm{C}, \mathrm{D}, \mathrm{F}, \mathrm{H}, \mathrm{I}, \mathrm{K}, \mathrm{L}, \mathrm{O})$ & $(\mathrm{A}, \mathrm{B}, \mathrm{C}, \mathrm{D}, \mathrm{F}, \mathrm{H}, \mathrm{I}, \mathrm{K}, \mathrm{L}, \mathrm{O})$ & $(\mathrm{A}, \mathrm{B}, \mathrm{C}, \mathrm{D}, \mathrm{F}, \mathrm{H}, \mathrm{I}, \mathrm{K}, \mathrm{L}, \mathrm{O})$ \\
\hline \multirow{2}{*}{0.3} & 41.38 & 40.38 & 39.38 & 40.38 & 41.38 \\
\hline & $(\mathrm{A}, \mathrm{B}, \mathrm{C}, \mathrm{D}, \mathrm{F}, \mathrm{H}, \mathrm{I}, \mathrm{K}, \mathrm{L}, \mathrm{O})$ & $(\mathrm{A}, \mathrm{B}, \mathrm{C}, \mathrm{D}, \mathrm{F}, \mathrm{H}, \mathrm{I}, \mathrm{K}, \mathrm{L}, \mathrm{O})$ & $(\mathrm{A}, \mathrm{B}, \mathrm{C}, \mathrm{D}, \mathrm{F}, \mathrm{H}, \mathrm{I}, \mathrm{K}, \mathrm{L}, \mathrm{O})$ & $(\mathrm{A}, \mathrm{B}, \mathrm{C}, \mathrm{D}, \mathrm{F}, \mathrm{H}, \mathrm{I}, \mathrm{K}, \mathrm{L}, \mathrm{O})$ & $(\mathrm{A}, \mathrm{B}, \mathrm{C}, \mathrm{D}, \mathrm{F}, \mathrm{H}, \mathrm{I}, \mathrm{K}, \mathrm{L}, \mathrm{O})$ \\
\hline \multirow{2}{*}{0.5} & 44.50 & 43.50 & 42.50 & 43.50 & 44.50 \\
\hline & $(\mathrm{A}, \mathrm{C}, \mathrm{D}, \mathrm{F}, \mathrm{H}, \mathrm{I}, \mathrm{K}, \mathrm{L}, \mathrm{M}, \mathrm{O})$ & $(\mathrm{A}, \mathrm{C}, \mathrm{D}, \mathrm{F}, \mathrm{H}, \mathrm{I}, \mathrm{K}, \mathrm{L}, \mathrm{M}, \mathrm{O})$ & $(\mathrm{A}, \mathrm{C}, \mathrm{D}, \mathrm{F}, \mathrm{H}, \mathrm{I}, \mathrm{K}, \mathrm{L}, \mathrm{M}, \mathrm{O})$ & $(\mathrm{A}, \mathrm{C}, \mathrm{D}, \mathrm{F}, \mathrm{H}, \mathrm{I}, \mathrm{K}, \mathrm{L}, \mathrm{M}, \mathrm{O})$ & $(\mathrm{A}, \mathrm{C}, \mathrm{D}, \mathrm{F}, \mathrm{H}, \mathrm{I}, \mathrm{K}, \mathrm{L}, \mathrm{M}, \mathrm{O})$ \\
\hline \multirow{2}{*}{0.7} & 47.20 & 46.40 & 45.60 & 46.40 & 47.20 \\
\hline & $(\mathrm{A}, \mathrm{C}, \mathrm{D}, \mathrm{E}, \mathrm{H}, \mathrm{I}, \mathrm{K}, \mathrm{L}, \mathrm{M}, \mathrm{O})$ & $(\mathrm{A}, \mathrm{C}, \mathrm{D}, \mathrm{E}, \mathrm{H}, \mathrm{I}, \mathrm{K}, \mathrm{L}, \mathrm{M}, \mathrm{O})$ & $(\mathrm{A}, \mathrm{C}, \mathrm{D}, \mathrm{E}, \mathrm{H}, \mathrm{I}, \mathrm{K}, \mathrm{L}, \mathrm{M}, \mathrm{O})$ & $(\mathrm{A}, \mathrm{C}, \mathrm{D}, \mathrm{E}, \mathrm{H}, \mathrm{I}, \mathrm{K}, \mathrm{L}, \mathrm{M}, \mathrm{O})$ & $(\mathrm{A}, \mathrm{C}, \mathrm{D}, \mathrm{E}, \mathrm{H}, \mathrm{I}, \mathrm{K}, \mathrm{L}, \mathrm{M}, \mathrm{O})$ \\
\hline \multirow{2}{*}{0.9} & 51.05 & 50.25 & 49.45 & 50.25 & 51.05 \\
\hline & $(\mathrm{A}, \mathrm{B}, \mathrm{C}, \mathrm{D}, \mathrm{E}, \mathrm{H}, \mathrm{I}, \mathrm{K}, \mathrm{L}, \mathrm{O})$ & $(\mathrm{A}, \mathrm{B}, \mathrm{C}, \mathrm{D}, \mathrm{E}, \mathrm{H}, \mathrm{I}, \mathrm{K}, \mathrm{L}, \mathrm{O})$ & $(\mathrm{A}, \mathrm{B}, \mathrm{C}, \mathrm{D}, \mathrm{E}, \mathrm{H}, \mathrm{I}, \mathrm{K}, \mathrm{L}, \mathrm{O})$ & $(\mathrm{A}, \mathrm{B}, \mathrm{C}, \mathrm{D}, \mathrm{E}, \mathrm{H}, \mathrm{I}, \mathrm{K}, \mathrm{L}, \mathrm{O})$ & $(\mathrm{A}, \mathrm{B}, \mathrm{C}, \mathrm{D}, \mathrm{E}, \mathrm{H}, \mathrm{I}, \mathrm{K}, \mathrm{L}, \mathrm{O})$ \\
\hline
\end{tabular}

The optimal solutions have been derived from GAMS 24.0.1, which is a high-level modeling system for mathematical optimization. Furthermore, the personal computer with $2.60 \mathrm{GHz}$ Intel Core i53230M CPU, 6GB of RAM and Windows 8 64-bit Operating System has been applied.

According to the results of Table 3, in the same probability level with the lowest possibility level for possibility-based model and the highest possibility level for necessity-based model, the optimal solutions of both possibility and necessity-based models are same. For different probability levels $\{0.1,0.3,0.5,0.7,0.9\}$, the best optimal solutions for possibility, necessity and hybrid-based models are obtained in possibility level of $\eta=0.1, \eta=0.9$ and $\eta=0.5$, respectively.

The best optimal solution of possibility, necessity and hybrid-based models are obtained as follows:

Possibility-based model: $\{\lambda=0.1, \eta=0.1\} \Rightarrow Z^{*}=32.76$ with optimal locations of (A, C, D, F, H, I, K, L, M, O),

Necessity-based model: $\{\lambda=0.1, \eta=0.9\} \Rightarrow Z^{*}=32.76$ with optimal locations of (A, C, D, F, H, I, K, L, M, O),

Hybrid-based model: $\quad\{\lambda=0.1, \eta=0.5\} \Rightarrow Z^{*}=34.76$ with optimal locations of (A, B, C, D, F, H, I, K, L, O), where these results are shown in Fig. 3 and Fig. 4 (red stars depict the optimal locations). 


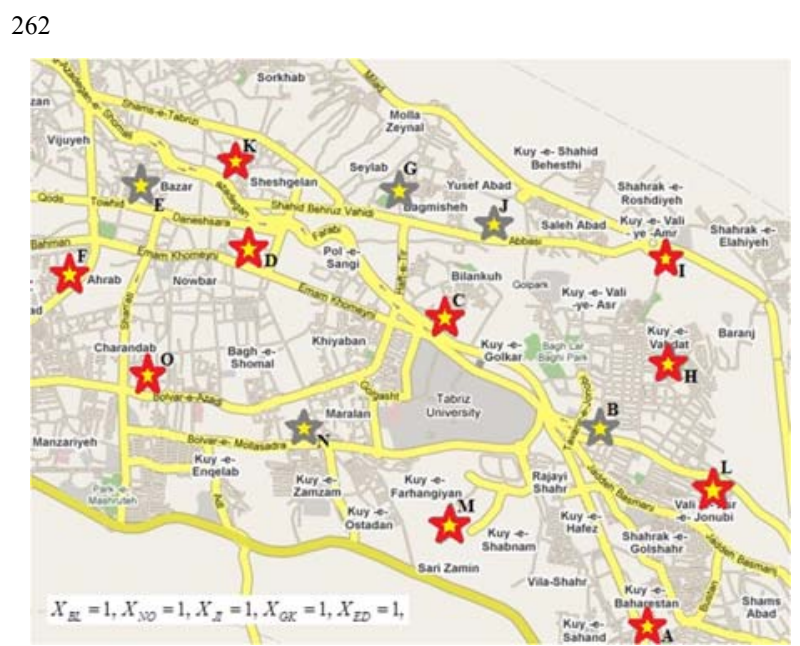

Fig. 3. Optimal locations of ten stations by possibility and necessity-based models

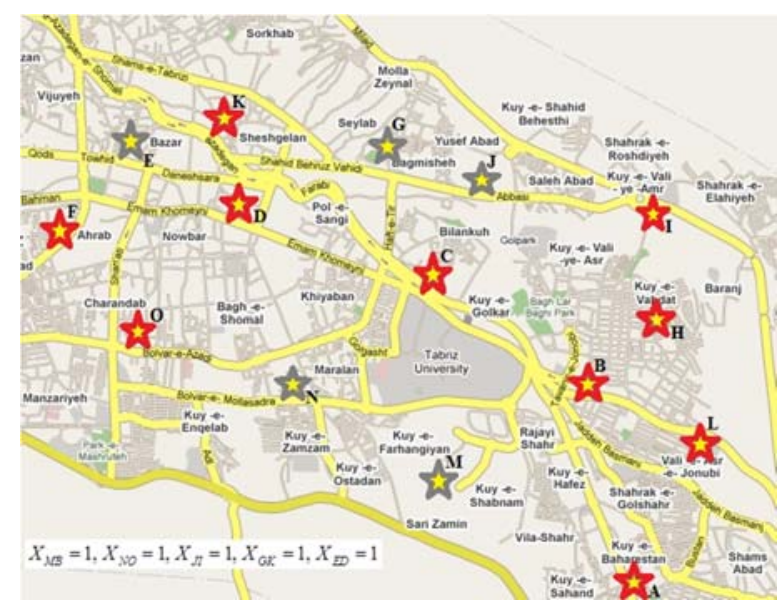

Fig. 4. Optimal locations of ten stations by hybridbased model

Fig. 3 and Fig. 4 indicate the best optimal solutions of the bicycle stations in Tabriz city in which these solutions are depend on the levels of probability and possibility and these levels are chosen by a DM's opinion. Based on the Table 3, 75 optimal solutions are obtained through possibility, necessity and hybrid-based models with different levels. Fig. 3 and Fig. 4 show the best optimal solutions of problem with specific levels of probability and possibility, but the DM can choose other levels based on his/her circumstances or any other constraints. Therefore, the DM's opinion can be categorized as follows:

a) The best optimal solution: this view of the DM is the same as the results have been depicted in Fig. 3 and Fig. 4. In this view, the DM searches for the best optimal solution with any levels of probability and possibility to allocate the bicycle stations and there is no limitation for choosing these levels.

b) The lowest or highest levels: this view shows that the DM wants to have the lowest or highest levels to allocate the bicycle stations. Therefore, he/she may encounter with these results:

The lowest levels:

Possibility optimal solution: $\{\lambda=0.1, \eta=0.1\} \Rightarrow Z^{*}=32.76$ with locations of (A, C, D, F, H, I, K, L, M, O),

Necessity optimal solution: $\quad\{\lambda=0.1, \eta=0.1\} \Rightarrow Z^{*}=36.76$ with locations of (A, B, C, D, F, H, I, K, L, O),

Hybrid optimal solution: $\quad\{\lambda=0.1, \eta=0.1\} \Rightarrow Z^{*}=36.76$ with locations of (A, B, C, D, F, H, I, K, L, O).

The highest levels:

Possibility optimal solution: $\{\lambda=0.9, \eta=0.9\} \Rightarrow Z^{*}=51.05$ with locations of (A, B, C, D, E, H, I, K, L, O)

Necessity optimal solution: $\quad\{\lambda=0.9, \eta=0.9\} \Rightarrow Z^{*}=47.85$ with locations of (A, C, D, E, H, I, K, L, M, O),

Hybrid optimal solution: $\quad\{\lambda=0.9, \eta=0.9\} \Rightarrow Z^{*}=51.05$ with locations of (A, B, C, D, E, H, I, K, L, O).

c) The Middle Levels: in this view, DM wants to have middle levels. This view happens when the DM does not have absolute information about the levels and decides to have middle levels. Therefore, the results based on this view are given as follows for all models:

$$
\{\lambda=0.5, \eta=0.5\} \Rightarrow Z^{*}=42.50 \text { with locations of (A, C, D, F, H, I, K, L, M, O). }
$$


In these levels, possibility, necessity and hybrid optimal solutions are the same and by this view, optimistic and pessimistic approaches for allocation problem will be disappeared and the DM can obtain same optimal solutions by choosing these levels. Fig. 5, Fig. 6 and Fig. 7 show the categories of (b) and (c) clearly.

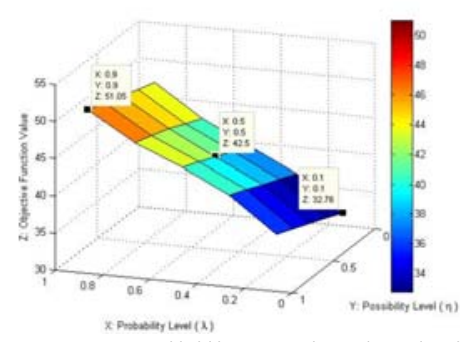

Fig. 5. Possibility optimal solutions for lowest, middle and highest levels of $\lambda, \eta$

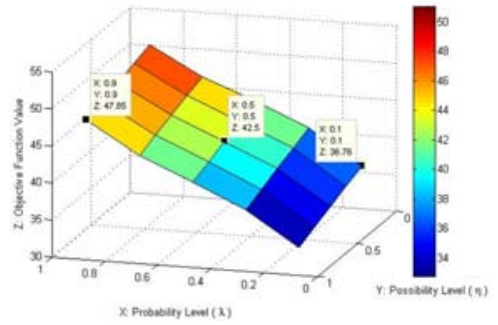

Fig. 6. Necessity optimal solutions for lowest, middle and highest levels of $\lambda, \eta$

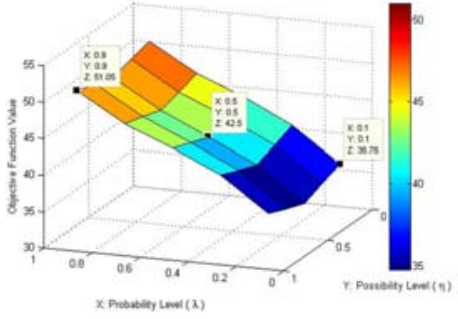

Fig. 7. hybrid optimal solutions for lowest, middle and highest levels of $\lambda, \eta$

It can be observed that the levels of $\{0.1,0.3,0.5,0.7,0.9\}$ indicated in this case study were sample levels to obtain the optimal solutions, so there is no limitation for the DM to choose only them to find the optimal solutions. One can choose any other levels between the ranges of $(0,1]$ to find his/her optimal solutions. Fig. 8, Fig. 9 and Fig. 10 indicate that any levels can be chosen by the DM to allocate the bicycle stations.

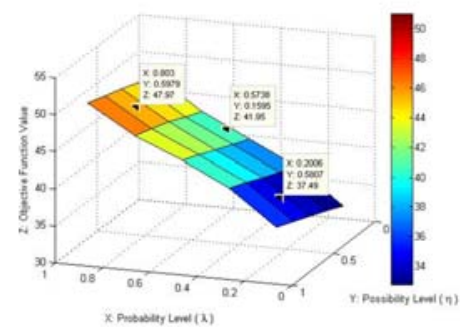

Fig. 8. Possibility optimal solutions for any levels of $\lambda, \eta$

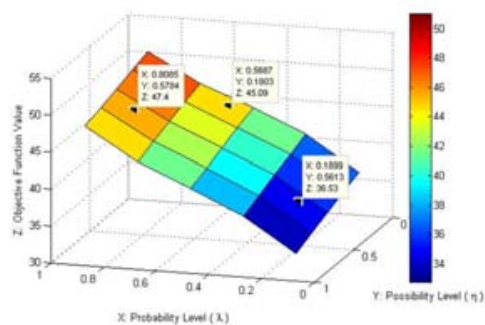

Fig. 9. Necessity optimal solutions for any levels of $\lambda, \eta$

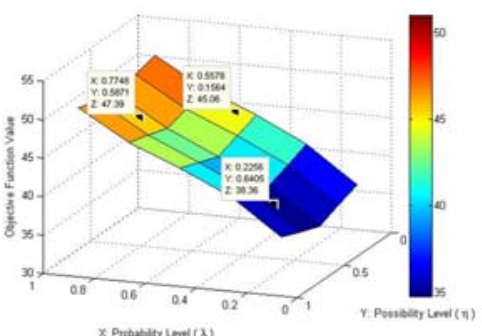

Fig. 10. Hybrid optimal solutions for any levels of $\lambda, \eta$

Furthermore, the DM may decide to solve the problem when the demand nodes are unweighted and consider the problem as a common vertex $p$-center problem without demand-weighted distance (constraint (14) is changed to $Z \geq \sum_{j} d_{i j} X_{i j} ; \forall i$ ). For this case study, by solving this common vertex $p$ center problem through GAMS 24.0.1, the optimal locations are derived as (A, C, D, G, H, I, J, M, N, $\mathrm{O})$ which is different from the best optimal solutions of possibility, necessity and hybrid-based models.

The obtained solution of the common vertex $p$-center problem is not suitable for this case study, because in allocation of the bicycle stations, the demand at node $i$ is an essential parameter and it is not rational to consider this parameter unweighted in the original problem. In other words, in the common vertex $p$-center problem, all demand nodes are treated equally and their weights are considered as one and because of the nature of allocating the bicycle stations in which the demand parameter is a necessary element, this notion is not absolutely correct. For example, perhaps a node which is one of the optimal locations has less demand than another node which has higher demand and has not been chosen as the optimal solution and it is in conflict with the nature of our case in which the optimal locations should be chosen in nodes with high demand. Therefore, the optimal locations obtained from common vertex $p$-center problem are not useful for allocating the bicycle stations. Also in the realworld situation, the exact value of this parameter is unavailable and has uncertain properties. Therefore, 
the results of this case study through possibility, necessity and hybrid-based models which were dealing with FRVs show the efficiency of the proposed methods and are more reliable for the DM to allocate the bicycle stations rather than considering the common vertex $p$-center problem.

\section{Conclusions}

In this paper, the vertex p-center problem with fuzzy random demand-weighted distance was introduced. Then, new methods were proposed to solve the problem to optimistic and pessimistic DMs separately and simultaneously, using the FRCCP based on the possibility theory. Finally, by using a case study about locations of the bicycle stations in Tabriz city, the results were calculated by possibility, necessity and hybrid-based models to compare these results with each other and to illustrate the deference between optimistic and pessimistic DMs. Furthermore, obtained models were compared with the common vertex p-center problem.

Because of applying demand node by FRVs in which variance effects, probability and possibility distribution function of parameters were considered, proposed methods are more useful. As these results showed in this case study, our fuzzy random model is robust and can be applied to the real cases with uncertainty. As suggestions for future research, the proposed methods can be used for other facility location problems with uncertain frameworks like hub-location, p-median, etc.

\section{Appendix A}

Proof of theorem 1:

I) Suppose that $\omega \in \Omega$ then

$$
\begin{aligned}
& \operatorname{Pos}\left(\mathrm{Z} \geq \tilde{h}_{i}(\omega) \sum_{\mathrm{j}} \mathrm{d}_{\mathrm{ij}} X_{i j}\right) \geq \eta \\
& \Leftrightarrow \sup _{y_{1}, y_{2} \in R}\left\{\min \left\{\mu_{Z}\left(y_{1}\right), \mu_{\hat{h}_{i}(\omega)} \sum_{\mathrm{j}} \mathrm{d}_{\mathrm{ij}} X_{i j}\left(y_{2}\right)\right\} \mid y_{1} \geq y_{2}\right\} \geq \eta \\
& \Leftrightarrow \quad \exists y_{1}, y_{2}\left(y_{1} \geq y_{2}\right): y_{1}=Z, L\left(\frac{h_{i}^{0}(\omega) \sum_{\mathrm{j}} \mathrm{d}_{\mathrm{ij}} X_{i j}-y_{2}}{\beta_{i} \sum_{\mathrm{j}} \mathrm{d}_{\mathrm{ij}} X_{i j}}\right) \geq \eta, R\left(\frac{y_{2}-h_{i}^{1}(\omega) \sum_{\mathrm{j}} \mathrm{d}_{\mathrm{ij}} X_{i j}}{\gamma_{i} \sum_{\mathrm{j}} \mathrm{d}_{\mathrm{ij}} X_{i j}}\right) \geq \eta \\
& \Leftrightarrow \quad \exists y_{1}, y_{2}\left(y_{1} \geq y_{2}\right): y_{1}=Z, h_{i}^{0}(\omega) \sum_{\mathrm{j}} \mathrm{d}_{\mathrm{ij}} X_{i j}-L^{*}(\eta) \beta_{i} \sum_{\mathrm{j}} \mathrm{d}_{\mathrm{ij}} X_{i j} \leq y_{2} \leq h_{i}^{0}(\omega) \sum_{\mathrm{j}} \mathrm{d}_{\mathrm{ij}} X_{i j}+R^{*}(\eta) \gamma_{i} \sum_{\mathrm{j}} \mathrm{d}_{\mathrm{ij}} X_{i j} \\
& \Leftrightarrow \quad Z \geq h_{i}^{0}(\omega) \sum_{\mathrm{j}} \mathrm{d}_{\mathrm{ij}} X_{i j}-L^{*}(\eta) \beta_{i} \sum_{\mathrm{j}} \mathrm{d}_{\mathrm{ij}} X_{i j}
\end{aligned}
$$

Let $h_{i}^{(2)} \sum_{j} \mathrm{~d}_{\mathrm{ij}} X_{i j}$ be positive, by applying the definition of $h_{i}^{0}(\omega)$, we have the following relations:

$$
\begin{aligned}
& \operatorname{Pr}\left\{\omega \mid \operatorname{Pos}\left(\mathrm{Z} \geq \tilde{h}_{i}(\omega) \sum_{\mathrm{j}} \mathrm{d}_{\mathrm{ij}} X_{i j}\right) \geq \eta\right\} \geq \lambda \\
& \Leftrightarrow \operatorname{Pr}\left\{\omega \mid t(\omega) \leq \frac{Z-\left(h_{i}^{0}-L^{*}(\eta) \beta_{i}\right) \sum_{\mathrm{j}} \mathrm{d}_{\mathrm{ij}} X_{i j}}{h_{i}^{(2)} \sum_{\mathrm{j}} \mathrm{d}_{\mathrm{ij}} X_{i j}}\right\} \geq \lambda \\
& \Leftrightarrow T\left(\frac{Z-\left(h_{i}^{0}-L^{*}(\eta) \beta_{i}\right) \sum_{\mathrm{j}} \mathrm{d}_{\mathrm{ij}} X_{i j}}{h_{i}^{(2)} \sum_{\mathrm{j}} \mathrm{d}_{\mathrm{ij}} X_{i j}}\right) \geq \lambda
\end{aligned}
$$

Since the function $T$ is not decreasing, 


$$
\begin{aligned}
& \Leftrightarrow \frac{Z-\left(h_{i}^{0}-L^{*}(\eta) \beta_{i}\right) \sum_{\mathrm{j}} \mathrm{d}_{\mathrm{ij}} X_{i j}}{h_{i}^{(2)} \sum_{\mathrm{j}} \mathrm{d}_{\mathrm{ij}} X_{i j}} \geq T^{*}(\lambda) \\
& \Leftrightarrow \quad Z \geq\left(h_{i}^{(0)}+\mathrm{T}^{*}(\lambda) h_{i}^{(2)}-L^{*}(\eta) \beta_{i}\right) \sum_{\mathrm{j}} \mathrm{d}_{\mathrm{ijj}} X_{i j} .
\end{aligned}
$$

\section{Appendix B}

\section{Proof of theorem 2:}

I) Let $\omega \in \Omega$ then

$$
\begin{aligned}
& \operatorname{Nec}\left(Z \geq \tilde{h}_{i}(\omega) \sum_{\mathrm{j}} \mathrm{d}_{\mathrm{ij}} X_{i j}\right) \geq \eta \\
& \Leftrightarrow \inf _{y_{1}, y_{2} \in R}\left\{\max \left\{1-\mu_{Z}\left(y_{1}\right), 1-\mu_{\hat{h}_{i}(\omega)} \sum_{\mathrm{j}} \mathrm{d}_{\mathrm{j}} X_{i j}\left(y_{2}\right)\right\} \mid y_{1} \geq y_{2}\right\} \geq \eta \\
& \Leftrightarrow \quad \forall y_{1}, y_{2}\left(y_{1} \geq y_{2}\right): y_{1}=Z \Rightarrow 1-\mu_{\hat{h}_{i}(\omega) \sum_{\mathrm{j}} \mathrm{d}_{\mathrm{j}} X_{i j}}\left(y_{2}\right) \geq \eta \\
& \Leftrightarrow \quad \forall y_{1}, y_{2}\left(y_{1} \geq y_{2}\right): y_{1}=Z \Rightarrow y_{2} \leq h_{i}^{0}(\omega) \sum_{\mathrm{j}} \mathrm{d}_{\mathrm{ij}} X_{i j}-L^{*}(1-\eta) \beta_{i} \sum_{\mathrm{j}} \mathrm{d}_{\mathrm{ij}} X_{i j} \vee \\
& \qquad y_{2} \geq h_{i}^{1}(\omega) \sum_{\mathrm{j}} \mathrm{d}_{\mathrm{ij}} X_{i j}+R^{*}(1-\eta) \gamma_{i} \sum_{\mathrm{j}} \mathrm{d}_{\mathrm{ij}} X_{i j} \\
& \Leftrightarrow \quad Z \geq h_{i}^{0}(\omega) \sum_{\mathrm{j}} \mathrm{d}_{\mathrm{ij}} X_{i j}-L^{*}(1-\eta) \beta_{i} \sum_{\mathrm{j}} \mathrm{d}_{\mathrm{ij}} X_{i j}
\end{aligned}
$$

Let $h_{i}^{(2)} \sum_{\mathrm{j}} \mathrm{d}_{\mathrm{ij}} X_{i j}$ be positive. By applying the definition of $h_{i}^{0}(\omega)$, we have the following relations:

$$
\begin{aligned}
& \operatorname{Pr}\left\{\omega \mid \operatorname{Pos}\left(\mathrm{Z} \geq \tilde{h}_{i}(\omega) \sum_{\mathrm{j}} \mathrm{d}_{\mathrm{ij}} X_{i j}\right) \geq \eta\right\} \geq \lambda \Leftrightarrow \operatorname{Pr}\left\{\omega \mid t(\omega) \leq \frac{Z-\left(h_{i}^{0}-L^{*}(1-\eta) \beta_{i}\right) \sum_{\mathrm{j}} \mathrm{d}_{\mathrm{ij}} X_{i j}}{h_{i}^{(2)} \sum_{\mathrm{j}} \mathrm{d}_{\mathrm{ij}} X_{i j}}\right\} \geq \lambda \\
& \Leftrightarrow T\left(\frac{Z-\left(h_{i}^{0}-L^{*}(1-\eta) \beta_{i}\right) \sum_{\mathrm{j}} \mathrm{d}_{\mathrm{ij}} X_{i j}}{h_{i}^{(2)} \sum_{\mathrm{j}} \mathrm{d}_{\mathrm{ij}} X_{i j}}\right) \geq \lambda
\end{aligned}
$$

Since the cumulative distribution function $T$ is not decreasing,

$$
\begin{aligned}
& \Leftrightarrow \frac{Z-\left(h_{i}^{0}-L^{*}(1-\eta) \beta_{i}\right) \sum_{\mathrm{j}} \mathrm{d}_{\mathrm{ij}} X_{i j}}{h_{i}^{(2)} \sum_{\mathrm{j}} \mathrm{d}_{\mathrm{ij}} X_{i j}} \geq T^{*}(\lambda) \\
& \Leftrightarrow \quad Z \geq\left(h_{i}^{(0)}+\mathrm{T}^{*}(\lambda) h_{i}^{(2)}-L^{*}(1-\eta) \beta_{i}\right) \sum_{\mathrm{j}} \mathrm{d}_{\mathrm{ij}} X_{i j} .
\end{aligned}
$$

\section{References}

Albareda-Sambola, M., Díaz, J. A., \& Fernández, E. (2010). Lagrangean duals and exact solution to the capacitated p-center problem. European Journal of Operational Research, 201(1), 71-81.

Daskin, M. S. (2011). Network and discrete location: models, algorithms, and applications. John Wiley \& Sons.

Döyen, A., Aras, N., \& Barbarosoğlu, G. (2012). A two-echelon stochastic facility location model for humanitarian relief logistics. Optimization Letters, 6(6), 1123-1145.

Dubois, D., \& Prade, H. (2001). Possibility theory, probability theory and multiple-valued logics: A clarification. Annals of mathematics and Artificial Intelligence, 32(1-4), 35-66.

Farahani, R. Z., SteadieSeifi, M., \& Asgari, N. (2010). Multiple criteria facility location problems: A survey. Applied Mathematical Modelling, 34(7), 1689-1709. 
Hakimi, S. L. (1965). Optimum distribution of switching centers in a communication network and some related graph theoretic problems. Operations Research, 13(3), 462-475.

Ishii, H., Lee, Y. L., \& Yeh, K. Y. (2007). Fuzzy facility location problem with preference of candidate sites. Fuzzy Sets and Systems, 158(17), 1922-1930.

Kariv, O., \& Hakimi, S. L. (1979). An algorithmic approach to network location problems. I: The pcenters. SIAM Journal on Applied Mathematics, 37(3), 513-538.

Kim, Y., Lee, Y., \& Han, J. (2011). A splitter location-allocation problem in designing fiber optic access networks. European Journal of Operational Research, 210(2), 425-435.

Küçükdeniz, T., Baray, A., Ecerkale, K., \& Esnaf, Ş. (2012). Integrated use of fuzzy c-means and convex programming for capacitated multi-facility location problem. Expert Systems with Applications, 39(4), 4306-4314.

Kwakernaak, H. (1978). Fuzzy random variables-I. Definitions and theorems. Information Sciences, 15(1), 1-29.

Lu, C. C., \& Sheu, J. B. (2013). Robust vertex p-center model for locating urgent relief distribution centers. Computers \& Operations Research, 40(8), 2128-2137.

Murat, A., Verter, V., \& Laporte, G. (2011). A multi-dimensional shooting algorithm for the twofacility location-allocation problem with dense demand. Computers \& Operations Research, 38(2), 450-463.

Puri, M. L., \& Ralescu, D. A. (1986). Fuzzy random variables. Journal of Mathematical Analysis and Applications, 114(2), 409-422.

Revelle, C. S., Eiselt, H. A., \& Daskin, M. S. (2008). A bibliography for some fundamental problem categories in discrete location science. European Journal of Operational Research, 184(3), 817-848.

Schütz, P., Stougie, L., \& Tomasgard, A. (2008). Stochastic facility location with general long-run costs and convex short-run costs. Computers \& Operations Research, 35(9), 2988-3000.

Wang, S., Watada, J., \& Pedrycz, W. (2009). Value-at-Risk-based two-stage fuzzy facility location problems. IEEE Transactions on Industrial Informatics,5(4), 465-482.

Wang, S., \& Watada, J. (2012). A hybrid modified PSO approach to VaR-based facility location problems with variable capacity in fuzzy random uncertainty. Information Sciences, 192, 3-18.

Wang, S., \& Watada, J. (2013). Capacitated two-stage facility location problem with fuzzy costs and demands. International Journal of Machine Learning and Cybernetics, 4(1), 65-74.

Wen, M., \& Kang, R. (2011). Some optimal models for facility location-allocation problem with random fuzzy demands. Applied Soft Computing, 11(1), 1202-1207.

$\mathrm{Xu}, \mathrm{D} ., \mathrm{Gao}, \mathrm{D} ., \quad \& \mathrm{Wu}, \mathrm{C}$. (2013). A primal-dual-approximation algorithm for the stochastic facility location problem with submodular penalties. Optimization, (ahead-of-print), 1-10. 